\title{
Raman Investigations of Metal Chalcogenide Thin Films (A Short Review)
}

\author{
HO SOONMIN \\ Centre for Green Chemistry and Applied Chemistry, INTI International University, \\ Putra Nilai, 71800, Negeri Sembilan, Malaysia. \\ *Corresponding author E-mail: soonmin.ho@newinti.edu.my \\ http://dx.doi.org/10.13005/ojc/35Specialissue101
}

(Received: December 26, 2018; Accepted: February 01, 2019)

\begin{abstract}
Preparation and characterization of thin films have been reported by many researchers. Because of thin films could be used in wide applications including optoelectronic, solar cells, laser devices and gas sensor. There are several tools have been employed to characterize the obtained materials such as structural, morphological, compositional, optical and electrical of samples. For example, X-ray diffraction, scanning electron microscopy, X-ray photoelectron spectroscopy, UV-Visible spectrophotometer, transmission microscopy and Fourier Transform Infrared spectroscopy as reported in literature review. In this work, Raman spectroscopy will be used to investigate the obtained films. Briefly discuss the advantages and disadvantages of this tool will be described also.
\end{abstract}

Keywords: Raman spectroscopy, Thin films, Band gap, Solar cells, Semiconductor.

\section{INTRODUCTION}

The binary ${ }^{1-8}$, ternary ${ }^{9-15}$, quaternary ${ }^{16-18}$ and penternary compounds ${ }^{19-22}$ have received great attention owning to their unique properties. These films are used in a wide variety of applications such as solar cell ${ }^{23-25}$, sensors ${ }^{26}$, photodiode arrays ${ }^{27}$, photoconductors ${ }^{28}$ and optoelectronic devices ${ }^{29}$. The chalcogen is the elements (oxygen, sulfur, selenium and tellurium) in Group 16 of the periodic table. The band gap energy was estimated to be in the range of $1-3 \mathrm{eV} .{ }^{30,31}$. Currently, the solar cell market is dominated by silicon solar cell ${ }^{32}$ due to being abundant, non-toxic ${ }^{33}$ and showingn remarkably higher power conversion efficiency. However, these solar cells are more expensive that other types of cells ${ }^{34}$. Thin film solar cell has always been cheaper but less efficient. This solar cell is favorable due to its minimum material usage. The two major thin film technologies such as cadmium telluride and copper indium gallium diselenide successfully contributed about $10 \%$ of the global production market share. The major drawback is a highly toxic material such as tellurium, cadmium ${ }^{35}$, and selenium.

This is an Open Access article licensed under a Creative Commons license: Attribution 4.0 International (CC- BY). Published by Oriental Scientific Publishing Company @ 2018 
In this work, thin films were investigated using Raman spectroscopy. The purity of sample and the phase identification were also examined.

\section{Literature survey}

Raman spectroscopy provides useful information on molecular interactions ${ }^{36}$, crystallinity, crystal phase ${ }^{37}$ and chemical structure ${ }^{38}$. Raman is based upon the interaction of light with the chemical bonds within a material. Raman spectra exhibit the intensity of the scattered photons versus the frequency difference to the incident photons. Typically, the peaks fall within a range of 500 to $2000 \mathrm{~cm}^{-1}$ and only appear if vibrational modes are sensitive to the laser wavelength used ${ }^{39}$.

Raman spectroscopy is chemical analysis technique ${ }^{40}$ and is employed to measure the scattering radiation ${ }^{41}$ of a matter (Fig. 1). Generally, when light is scattered by matter, almost all of the scattering is an elastic process ${ }^{42}$ and does not give useful information (commonly known as Rayleigh Scatter). However, a very small percentage of scattering ${ }^{43}$ is an inelastic process (known as Raman effect). Raman spectroscopy is both qualitative (measuring the frequency ${ }^{44}$ of the scattered radiations) and quantitative (measurement of the analyte concentration in the sample by quantifying the intensity of the scattered radiations). The obtained Raman spectra give a unique data ${ }^{45,46}$ to identify a material and distinguish it from others. Table 1 shows the advantages and limitations of Raman spectroscopy technique.

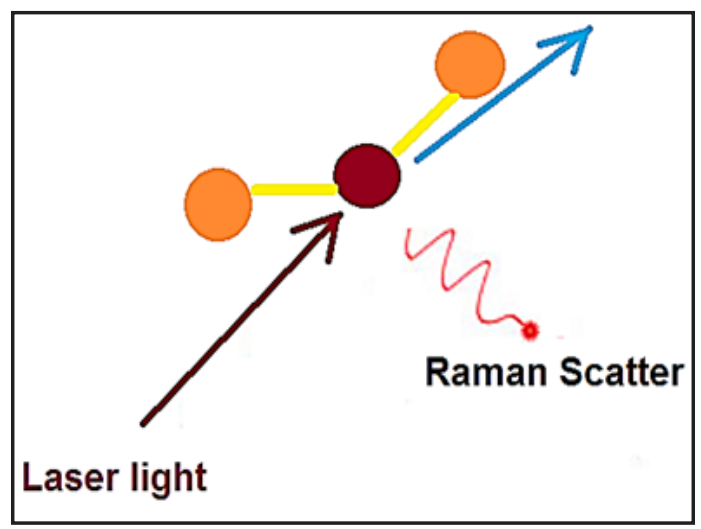

Fig. 1. Raman is a light scattering technique.

Several efforts have been made by researchers to synthesize semiconductor thin films such as sulfur, selenium and tellurium-based nanostructured films. Typical deposition technique is shown in Table 2. In this study, the utilization of Raman spectroscopy for the characterization of thin films wais discussed.

Table 1: Advantage and disadvantage of Raman spectroscopic

\begin{tabular}{|c|c|}
\hline Advantage & Disadvantage \\
\hline \multicolumn{2}{|l|}{ Advantage } \\
\hline \multicolumn{2}{|l|}{ Disadvantage } \\
\hline -Evaluation of trace element contents without the extensive & - It cannot be employed for alloys \\
\hline -Water is the best solvent to dissolve sample. & - The weak Raman scattering will lead to the low sensitivity. \\
\hline -Raman spectroscopy detected organic or inorganic materials ${ }^{48}$ & - The presence of impurities can hide Raman spectra \\
\hline -It is simplicity method & $\begin{array}{l}\text { - Heating through intense laser radiation can destroy the } \\
\text { Raman spectra }\end{array}$ \\
\hline \multicolumn{2}{|l|}{$\begin{array}{l}\text {-It can be used for in-field, in-situ }{ }^{49} \text { or down-field in the } \\
\text { range } 4000-50 \mathrm{~cm}^{-1}\end{array}$} \\
\hline •Raman spectroscopy allows quick identification of materials ${ }^{50}$ & \\
\hline
\end{tabular}

Table 2: Raman spectroscopic investigation of thin films prepared under various deposition conditions

\begin{tabular}{lll}
\hline Thin films & Raman spectroscopy explained & Deposition method \\
Binary thin films & Reference \\
\hline Thalium & $\cdot$ TISe ${ }_{2}$ bending mode at $92 \mathrm{~cm}^{-1}$ & Thermal \\
selenide & $\cdot$ TI-Se symmetric stretching modes at 158 and $140 \mathrm{~cm}^{-1}$ & evaporation \\
& $\cdot T l-S e$ asymmetric stretching modes at 208 and $185 \mathrm{~cm}^{-1}$ & med
\end{tabular}


-In terms of crystal structure, TI-Se bond was observed in 2.66-2.73 $\AA$

Arsenic triselenide $\left(\mathrm{As}_{2} \mathrm{Se}_{3}\right)$

-The peak at $224 \mathrm{~cm}^{-1}$ corresponded to asymmetric

(for amorphous phase)

-Weak peak at $480 \mathrm{~cm}^{-1}$ represented Se-Se vibration

-The peak at $221 \mathrm{~cm}^{-1}$ could be seen in In-doped $\mathrm{As}_{2} \mathrm{Se}_{3}$ films

-There are two peaks at $216-219 \mathrm{~cm}^{-1}$ and $240-242 \mathrm{~cm}^{-1}$ for

the films prepared with indium content of $2 \%$.

Antimony $\quad \cdot$ Peak at $188 \mathrm{~cm}^{-1}$ : Sb-Se stretching mode

Thermal

[52]

-Peak at $150 \mathrm{~cm}^{-1}: \mathrm{Sb}-\mathrm{Sb}$ bond

$\left(\mathrm{Sb}_{2} \mathrm{Se}_{3}\right) \quad$ Peak at 120 and $210 \mathrm{~cm}^{-1}$ : vibration mode Se-Se bond

-Raman spectra reflected selenium rich in sample

(peaks at 70, 102, 129, $252 \mathrm{~cm}^{-1}$ ).

Tungsten $\quad$ Peak at $175 \mathrm{~cm}^{-1}$ represented vibration mode

disulfide $\quad$ Peak at $419 \mathrm{~cm}^{-1}$ attributed to $W_{2}$ phase

$\left(\mathrm{WS}_{2}\right)$

$\mathrm{SnS}_{2}$ films

Pulsed laser deposition

evaporation

- Citric acid was used as complexing agent during the deposition process.

-The films prepared using $0.375,0.5$ and $0.625 \mathrm{ml} / \mathrm{L}$ citric acid indicated

the peak at $315 \mathrm{~cm}^{-1}$ (mode of hexagonal $\mathrm{SnS}_{2}$ phase).

-Researchers confirm the phase purity of sample.

Chemical bath deposition

SnS $\quad$ The peak was observed at $307 \mathrm{~cm}^{-1}$ for the films prepared using 40

and $50 \mathrm{mTorr}$, indicating that the formation of $\mathrm{Sn}_{2} \mathrm{~S}_{3}$.

-There are two peaks ( 93 and $224 \mathrm{~cm}^{-1}$ ) could be seen for the films

prepared using 6 and $10 \mathrm{mTorr}$, respectively.

-An additional small peak in the spectra $\left(135 \mathrm{~cm}^{-1}\right)$ was detected

for the films synthesized under 40 , and 50 mTorr.

RF sputtering

$\mathrm{FeS}_{2}$

-There are two main phases (pyrite [343, $\left.379 \& 430 \mathrm{~cm}^{-1}\right]$ and marcasite [323 \& $\left.386 \mathrm{~cm}^{-1}\right]$ ) of development for the synthesis of iron sulphide.

-Pyrite was preferentially produced (pyrite:marcasite about 99:1) at high temperature $\left(420^{\circ} \mathrm{C}\right)$.

-At low deposition temperature $\left(250^{\circ} \mathrm{C}\right)$, marcasite was grown preferentially.

InS

-There are very broad peaks at $200-500 \mathrm{~cm}^{-1}$ may indicate the presence

of amorphous or nano crystalline materials.

-Researchers were able to identify some peaks at $200-400 \mathrm{~cm}^{-1}$

contributed to the $\ln _{2} S_{3}$

-Peak at $460 \mathrm{~cm}^{-1}$ attributed to the S-S mode of sulphur

$\ln _{2} \mathrm{~S}_{3} \quad-$ There are 3 peaks $\left(115,135\right.$ and $\left.180 \mathrm{~cm}^{-1}\right)$ could be observed for the films prepared at 60,70 and $80^{\circ} \mathrm{C}$.

- The presence of $\ln _{2} \mathrm{O}_{3}$ could be detected for the films deposited at $60^{\circ} \mathrm{C}$

-The intensity of peak is improved in annealed films.

$\mathrm{As}_{2} \mathrm{~S}_{3} \quad \cdot$ There are 3 peaks $\left(336,230,485 \mathrm{~cm}^{-1}\right)$ could be seen for the

films prepared using laser light

-Peaks at $335-340 \mathrm{~cm}^{-1}$ associated with As-S bond stretching

vibration (pyramidal phase).

-Peaks at 180 and $230 \mathrm{~cm}^{-1}$ attributed to As-As homopolar bond vibration

-Peak at $485 \mathrm{~cm}^{-1}$ corresponded to S-S vibration of $\mathrm{AsS}_{4}$

CdTe Both the transverse $\left(142 \mathrm{~cm}^{-1}\right)$ and longitudinal $\left(170.5 \mathrm{~cm}^{-1}\right)$ mode could be found.

-Peak at $120 \mathrm{~cm}^{-1}$ attributed to phonon of tellurium

ZnS films - Two peaks at 773 and $1078 \mathrm{~cm}^{-1}$ were detected in annealed and as-deposited films.

-The lattice constant value for both as-deposited $(5.3 \AA \AA)$ and annealed films (5.66 Å) was determined.

$\mathrm{Bi}_{2} \mathrm{Te}_{3} \quad \cdot$ Peak at $77 \mathrm{~cm}^{-1}$ attributed to vibration mode of BiTe

-Peaks at 65 and $131 \mathrm{~cm}^{-1}$ due to vibration mode of trigonal direction

Electro

[56]

Evaporation

method

Chemical

bath

deposition

Chemical bath

deposition

Thermal evaporation

-Peak at $102 \mathrm{~cm}^{-1}$ corresponded to vibration mode in the basal plane

-Three peaks (299, 600 and $\left.900 \mathrm{~cm}^{-1}\right)$ were found and could be observed as longitudinal optical phonons. 
Ge-Sb-Se $\quad$ Four main modes such as symmetric stretching mode of $\mathrm{GeSe}_{4} / 2$ tetrahedral $\left(200,215 \mathrm{~cm}^{-1}\right)$, stretching mode of $\mathrm{Ge}-\mathrm{Ge}$ bond $\left(170 \mathrm{~cm}^{-1}\right)$ Se-Se stretching mode $\left(235-245 \mathrm{~cm}^{-1}\right)$ and Se-Se bond vibration $\left(265 \mathrm{~cm}^{-1}\right)$ are observed in these films

$\mathrm{Ge}_{2} \mathrm{Sb}_{2} \mathrm{Te}_{5}$ -They observe some peaks such as 80 (GeTe4 tetrahedral), 125 (GeTe4-nGen(n=1,2)), 153 (presence of Sb2Te3) and $300 \mathrm{~cm}^{-1}$ (presence of $\mathrm{Ge}-\mathrm{Ge}$ ) in spectra

$\mathrm{Ge}_{\mathrm{x}} \mathrm{As}_{35-\mathrm{x}} \mathrm{Se}_{65} \quad$ The films exhibited symmetric vibrational stretching $\mathrm{GeSe}_{4} / 2$ at $198 \mathrm{~cm}^{-1}$, vibration mode of $\mathrm{AsSe}_{3} / 2$ at $230 \mathrm{~cm}^{-1}$, vibration mode of selenium at 260 $\mathrm{cm}^{-1}$ and vibration mode of $\mathrm{GeSe}_{4} / 2$ tetrahedral at $215 \mathrm{~cm}^{-1}$

$\mathrm{As}_{50} \mathrm{Se}_{40} \mathrm{Te}_{10} \quad \cdot$ Three major modes such as asymmetric stretching As-Te-Se mode $\left(127 \mathrm{~cm}^{-1}\right)$, As-Se vibration mode $\left(228 \mathrm{~cm}^{-1}\right)$ and Te-Te vibrational mode $\left(472 \mathrm{~cm}^{-1}\right)$ are observed in these films

$\mathrm{Cu}_{2} \mathrm{SnS}_{3} \quad \cdot$ The Raman peaks exhibit strong (vibration mode in monoclinic) and weak peak $\left(\mathrm{Cu}_{2} \mathrm{SnS}_{3}\right.$ phase).

$\mathrm{CuSbSe}_{2} \quad \cdot$ They observe several peaks such as $188,250,372,450 \mathrm{~cm}^{-1}$ $\left(\mathrm{Sb}_{2} \mathrm{Se}_{3}\right), 185 \mathrm{~cm}^{-1}\left(\mathrm{Cu}_{3} \mathrm{SbSe}_{3}\right)$, and $82,117,144 \mathrm{~cm}^{-1}$ (presence of $\mathrm{CuSbSe}_{2}$ ) in spectra

$\mathrm{Cu}_{12} \mathrm{Sb}_{4} \mathrm{~S}_{13} \quad$ There are many peaks such as $351 \mathrm{~cm}^{-1}\left(\mathrm{Cu}_{12} \mathrm{Sb}_{4} \mathrm{~S}_{13}\right), 354 \mathrm{~cm}^{-1}$ (Sb-S bond stretching), $315 \mathrm{~cm}^{-1}$ (Sb-S bond bending mode), $330 \mathrm{~cm}^{-1}\left(\mathrm{Cu}_{3} \mathrm{SbS}_{4}\right), 468 \mathrm{~cm}^{-1}$ (CuS) could be confirmed.

$\mathrm{Cu}_{2} \mathrm{SnS}_{3} \quad$ There are several peaks could be identified such as 290 and $350 \mathrm{~cm}^{-1}$ (monoclinic), $325 \mathrm{~cm}^{-1}$ (tetragonal phase), 223 and $371 \mathrm{~cm}^{-1}\left(\mathrm{Cu}_{2} \mathrm{Sn}_{3} \mathrm{~S}_{7}\right)$

$\mathrm{Cu}_{2} \mathrm{SnS}_{3} \quad \cdot$ The films indicated orthorhombic $\mathrm{Cu}_{3} \mathrm{SnS}_{4}$ phase at $295 \mathrm{~cm}^{-1}$, monoclinic $\mathrm{Cu}_{2} \mathrm{SnS}_{3}$ at $289 \mathrm{~cm}^{-1}$ and vibration mode of tetragonal $\mathrm{Cu}_{2} \mathrm{SnS}_{3}$ in anneal films at $326 \mathrm{~cm}^{-1}$

$\mathrm{Cu}_{2} \mathrm{SnSe}_{3} \quad \cdot$ Peak at $2926 \mathrm{~cm}^{-1}$ was observed (attributed to $\mathrm{C}-\mathrm{H}$ stretching vibration of $\mathrm{CH}_{2}$ ) for the films prepared using $0.5 \mathrm{M}$ of sodium citrate. -However, this peak could not be detected when using higher concentration $(0.1 \mathrm{M})$.

Quaternary thin films

$\mathrm{Cu}_{2} \mathrm{ZnSnS}_{4} \quad$ The films showed a prominent kesterite phase $\left(331-336 \mathrm{~cm}^{-1}\right)$, indicating microwave treatment enhanced nucleation and grow process.

$\mathrm{Cu}_{2} \mathrm{ZnSnS}_{4} \quad$ There is no Raman peak could be detected for the films prepared at room temperature and within the first 15 minutes.

-The films prepared under 30 min of sulfurization show peak at $330 \mathrm{~cm}^{-1}$ -The films synthesized under 60 and 180 min of sulfurization show higher intensities if compared to other conditions. -There are no other impurities ( $\mathrm{ZnS}, \mathrm{SnS}_{2}$ and $\mathrm{CuS}$ ) indicating high purity of sample.

$\mathrm{Cu}_{2} \mathrm{FeSnS}_{4} \quad$ Two main modes such as sulfur pure anion around the copper metal $\left(285 \mathrm{~cm}^{-1}\right)$ and asymmetry vibration mode of sulphur around the tin

CuGaxln $\mathrm{Se}_{1-\mathrm{x}} \mathrm{Se}_{2} \cdot$ The presence of $\mathrm{Cu}_{2} \mathrm{Se}\left(290 \mathrm{~cm}^{-1}\right)$ and $\mathrm{Cu}(\mathrm{In}, \mathrm{Ga}) \mathrm{Se}_{2}$ phase $\left(175 \mathrm{~cm}^{-1}\right)$ could be detected when $x=1$, and $x=0,0.3$, respectively.

$\mathrm{Cu}_{2} \mathrm{ZnSnS}_{4} \quad \bullet$ Kesterite phase $\left(332,285,356\right.$ and $\left.368 \mathrm{~cm}^{-1}\right)$, copper tin sulphide $\left(303 \mathrm{~cm}^{-1}\right)$ and copper sulphide $\left(468 \mathrm{~cm}^{-1}\right)$ were observed in Raman spectra.

Culn(S,Se $)_{2} \quad-$ There are many peaks at $473 \mathrm{~cm}^{-1}$ (Cu2-xS), 390-475 $\mathrm{cm}^{-1}$ (sulfur rich $\left.\mathrm{Cu}_{2-x}(\mathrm{~S}, \mathrm{Se})\right), 228 \mathrm{~cm}^{-1}\left(\mathrm{Culn}_{3} \mathrm{Se}_{5}\right)$ and $150-400 \mathrm{~cm}^{-1}$ (amorphous $\ln _{2} \mathrm{~S}_{3}$ ) could be confirmed.

deposition
Chemical bath

deposition

Pulsed laser
deposition

RF magnetron

sputtering

[65]

Thermal

evaporation

Thermal

evaporation

Thermal

evaporation

Evaporation

method

E-beam

evaporation

deposition

e-beam

evaporation

Radio frequency

magnetron sputtering

Spray pyrolysis

Electro deposition

[76]

Sol gel method

Sol gel technique

[78]

Spray pyrolysis

Close spaced

vapor transport technique

Spray pyrolysis

Chemical bath 
Penternary thin films

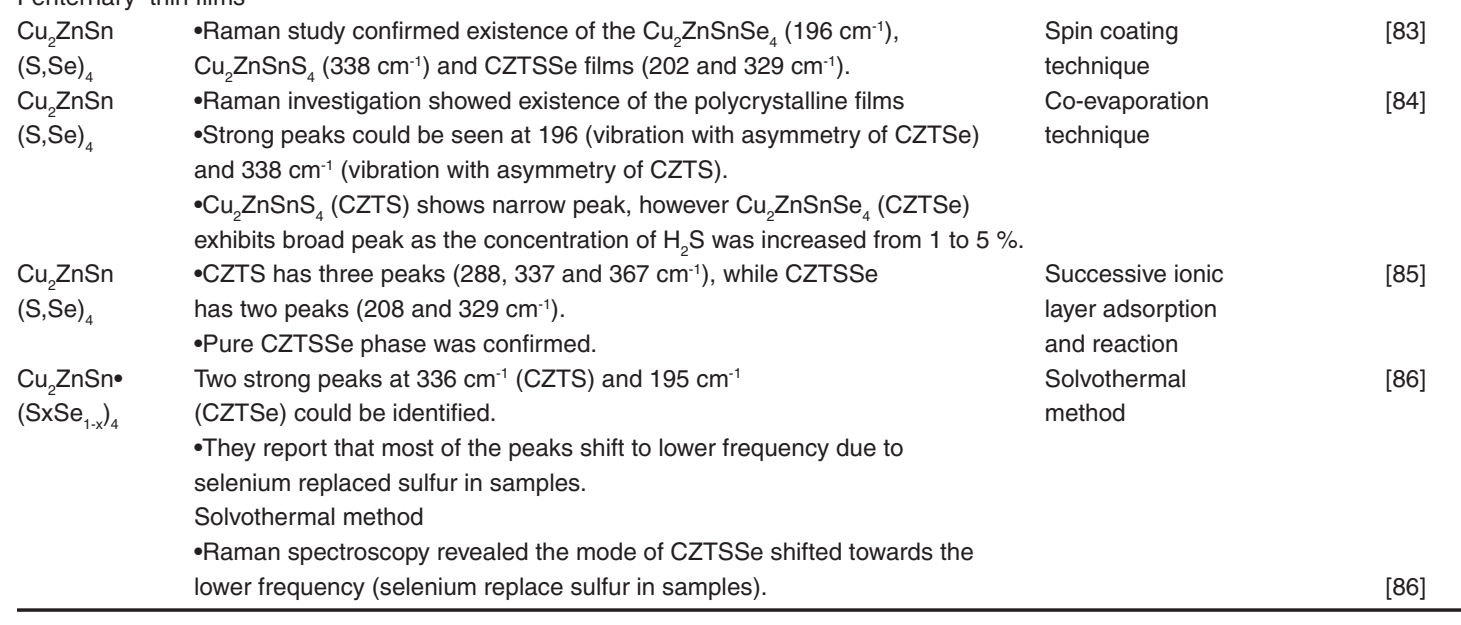

CONCLUSION

Raman spectroscopy is a powerful technique for characterization of thin films. Here, the author reviews the Raman spectra of nanostructured films. Also, the author indicates how to use it to determine structure and composition of samples. The purity of the compound and phase formation can be verified by this technique.

\section{ACKNOWLEDGEMENT}

Inti International University is gratefully acknowledged for the financial support of this work.

\section{Conflict of interest}

Author has declared that no competing interests exist.

\section{REFERENCES}

1. Khadher, A.; Mazahar, F.; Gulam, R. Orient. J. Chem., 2018, 34, 2203-2207.

2. Mohd, J.H.; Mohd, Y.R.; Ho, S.M.; Anuar, K.; Abdul, H.A.; Saravanan, N. Kuwait J. Sci. Eng., 2010, 37, 63-73.

3. Gao, C.; Xu, M.; Ng, B.K.; Kang, L.; Jiang, L.; Lai, Y.; Liu, F. Mater. Lett., 2017, 195, 186-189.

4. Tan, W.T.; Ho, S.M.; Anuar, K., Saravanan, N. Eur. J. Appl. Sci., 2011, 3, 75-80.

5. Shanmugasundaram, K.; Thirunavukkarasu, P.; Ramamurthy, M.; Balaji, M.; Chandrasekaran, J. Orient. J. Chem., 2017, 33, 2484-2491.

6. Victor, R.; Juan, F.T.; Cecilia, G.; Jose, H. Thin Solid Films., 2015, 582, 249-252.

7. Kassim, A.; Ho, S.M.; Tan, W.T.; Shanthi, M.; Saravanan, N. Kathmandu Univ. J. Sci. Eng. Technol., 2010, 6, 126-132.

8. Sakthivel, R.; Ganesh, A.S.; Geetha, A.; Anandh, B.; Kannusamy, R.; Tamilselvan, K. Orient. J. Chem., 2017, 33, 355-362.

9. Meena, S.P.; Ashokkumar, R. Orient. J. Chem., 2018, 34, 1884-1889.

10. Sarwan, B.; Aman, D.A. Orient. J. Chem.,
2018, 34, 2667-2670.

11. Soepardjo, A.H. Makara Sains, 2009, 13, 200-204.

12. Kanakaraj, M.; Ramalingam, H.B. Orient. J. Chem., 2017, 33, 2899-2904.

13. Anuar, K.; Saravanan, N.; Ho, S.M.;Tan, W.T.; Atan, S.; Zulkefly, K. Chiang Mai Univ. J. Nat. Sci., 2008, 7, 317-326.

14. Chalapathi, U.; Poornaprakash, B.; Park, S. Ceram. Int., 2017, 43, 5229-5235.

15. Ho, S.M. Orient. J. Chem., 2014, 30, 1009-1012.

16. Li, X.; Cao, H.; Dong, Y.; Yue, F.; Chen, Y.; Xiang, P.; Sun, L.; Yang, P.; Chu, J. J. Alloys Compd., 2017, 694, 833-840.

17. Soumyo, C.; Amlan, J. P. Sol. Energy Mater. Sol. Cells., 2017, 160, 233-240.

18. Dong, L.; Cheng, S.; Lai, Y.; Zhang, H.; Jia, H. Thin Solid Films., 2017, 626, 168-172.

19. Jakapan, C.; Takuya, K.; Hiroki, S.; Takashi, M. Curr. Appl. Phys., 2017, 17, 461-466.

20. Gang, M.G.; Shin, S.W.; Hong, C.W.; Gurav, K.V.; Gwak, J.; Yun, J.H.; Lee, J.Y.; Kim, J. H. Green Chem., 2016, 18, 700-711. 
21. Yang, K.; Sim, J.; Son, D.; Kim, Y.; Kim, D.; Nam, D.; Cheong, H.; Kim, S.; Kim, J.; Kang, J. Nano Energy., 2017, 35, 52-61.

22. Lv, X.; Wang, J.; Zhu, C.; Wang, Z. Rare Metals., 2016, 35, 718-722.

23. Wie, W.; Mark, T.W.; Oki, G.; Tayfun, G.; Teodor, K.T.; Yu, Z.; David, B.M. Adv. Energy Mater., 2014, https://doi.org/10.1002/ aenm.201301465.

24. Kassim, A.; Ho, S.M.; Saravanan, N.; Noraini, K. Arabian J. Chem., 2010, 3, 243-249.

25. Ngai, C.F.; Anuar, K.; Ho, S.M.; Tan, W.T. Am. J. Appl. Sci., 2011, 8, 359-361.

26. Ogita, M.; Higo, K.; Nakanishi, Y.; Hatanaka, Y. Appl. Surf. Sci., 2001, 175-176, 721-725.

27. Saxena, R.S.; Aparna, S. Infrared Phys. Technol., 2014, 65, 51-53.

28. Ren, Y.X.; Dai, T.J.; Luo, W.B.; Liu, X.Z. Vacuum., 2018, 149, 190-194.

29. Hosein, K. Ceram. Int., 2019, 45, 334-345.

30. Ashwini, B.R.; Priyanka, U.L.; Jeong, I.H.; Nandu, B. C. Appl. Surf. Sci., 2019, 466, 358-366.

31. Seyed, M.M.; Hosein, K. Superlattices Microstruct., 2019, 126, 139-149.

32. Green, M.A.; Basore, P.A.; Chang, N.; Clugston, D.; Egan, R.; Hogg, D.; Jarnason, S. Sol. Energy., 2004, 77, 857-863.

33. Gavin, C.; Green, M.; Corkish, R.; Cho, Y.; Cho, E.; Jiang, C.; Thipwan, F. Thin Solid Films., 2006, 511-512, 654-662.

34. Saga, T. NPG Asia Mater., 2010, 2, 96-102.

35. Fahhad, A.; John, D.B.; Salhi, A.; Ahmed, A.; Kim, H.; Miller, R.D. Renew. Energy., 2011, 36, 2753-2758.

36. Amer, M.S. Raman spectroscopy for soft matter applications. John Wiley \& Sons Inc: New Jersey., 2009.

37. Challa, S. Raman spectroscopy for nanomaterials characterization. Springer Science \& Business Media: Berlin., 2012.

38. Mantz, R.A. Molten salts XIV: Proceedings of the International Symposium, The Electrochemical Society: New Jersey., 2006.

39. Zhang, S. Raman spectroscopy and its application in nanostructures. John Wiley \& Sons, Inc: New Jersey., 2012.

40. Ewen, S.; Dent, G. Modern Raman spectroscopy: A practical approach. John Wiley \& Sons, Inc: New Jersey., 2005.

41. Peter, L. Infrared and Raman spectroscopy: Principles and spectral interpretation. $2^{\text {nd }}$ edition, Elsevier: Amsterdam., 2018.

42. John, C.L. Rayleigh scattering and Raman spectroscopy, theory. Encyclopedia of Spectroscopy and Spectrometry, Elsevier: London., 2000.

43. Shadi, A.D.; Anna, F.; Chennupati, J. Semiconductor nanowires II: properties and applications. Elsevier: London., 2016.

44. John, R.F.; Kazuo, N. Introductory Raman spectroscopy. Academic Press Inc, New York., 1994.

45. Fasolato, C. Surface enhanced Raman spectroscopy for biophysical applications: using plasmonic nanoparticle assemblies. Springer: Berlin., 2018.

46. Alan, C. Biophysical chemistry, Royal Society of Chemistry: London., 2004.

47. Weber, W.H.; Merlin, R. Raman scattering in materials science, Springer-Verlag: Berlin., 2000.

48. High Pressure studies of crystalline materials. MDPI: Basel., 2018.

49. Ian, R.L.; Howell, E. Handbook of Raman spectroscopy: from the research laboratory to the process line. Marcel Dekker Inc: New York., 2001.

50. Sasic, S. Pharmaceutical applications of Raman spectroscopy. A John Wiley \& Sons, Inc: New Jersey., 2008.

51. Ozel, A.E.; Deniz, D.; Sefa, C.; Sahin, Y.; Binnur, K.; Sevim, A.; Kemal, U. Physica B: Condens Matter., 2017, 527, 72-77.

52. Yuriy, A.; Volodymyr, D.; Dmytro, S.; Vasyl, L.; Laroslav, G.; Vasyl, L.; Alexander, G.; Dietrich, R.T. Appl. Surf. Sci., 2019, 471, 943-949.

53. Shongalova, A.; Correia, M.R.; Teixeira, J.P.; Leitao, J.P.; Gonzalez, J.C.; Ranjbar, S.; Garud, S.; Vermang, B.; Cunha, J.; Salome, P.; Fernandes, P. Sol. Energy Mater. Sol. Cells., 2018, 187, 219-226.

54. Kun, T.; Tiwari, A.; Baskaran, K. Thin Solid Films., 2018, 668, 69-73.

55. Jing, L.; Yong, C.; Zhang, M. Mater. Sci. Forum., 2010, 663-665, 104-107.

56. Vinaya, K.A.; Shin, Y.; Kim, J. Superlattices Microstruct., 2018, 122, 253-261.

57. Walimbe, A.; Wertheim, A.; Ravi, A.; Kopas, C.; Saxena, A.; Singh, R.K.; Lehner, S.W.; John, D.; Makar, J.; Carpenter, R.W.; Buseck, P.R.; Newman, N. Thin Solid Films., 2019, 669, 49-55. 
58. Asenjo, B.; Guillen, C.; Chaparro, A.M.; Saucedo, E.; Bermudez, V.; Lincot, D.; Herrero, J.; Gutierrez, M.T. J. Phys. Chem. Solids., 2010, DOI: doi:10.1016/j.jpcs.2010.09.011.

59. Rodriguez, P.E.; Flores, F.D.; Cervantes, A.; Campos, E.; Cruz, J.; Mayen, S. Chalcogenide Lett., 2016, 13, 389-396.

60. Azhniuk, Y.; Dmytro, S.; Loya, V.; Iaroslav, G.; Vasyl, L.; Gomonnai, A.V.; Zahn, D. Appl. Surf. Sci., 2019, 467-468, 119-123.

61. Mitric, J.; Paunovic, N.; Mitric, M.; Vasic, B.; Ralevic, U.; Trajic, J.; Romcevic, M.; Dobrowolski, W.D.; Yahia, I.S.; Romcevic, N. Physica E Low Dimens Syst Nanostruct., 2018, 104, 64-70.

62. Keshav, R.; Mahesha, M.G. Mater. Res. Bull., 2018, 105, 360-367.

63. Thorat, J.B.; Mohite, S.V.; Bagade, A.A.; Shinde, T.J.; Fulari, V.J.; Rajpure, K.Y.; Shinde, N.S. Mater. Sci. Semicond. Process., 2018, 79, 119-126.

64. Oladeji, I.O.; Chow, L.; Liu, J.R.; Chu, W.K.; Bustamante, A.; Fredricksen, C.; Schulte, A.F. Thin Solid Films., 2000, 359, 154-159.

65. Kariper, A.; Guneri, E.; Gode, F.; Gumus, C. Chalcogenide Lett., 2012, 9, 27-40.

66. Erazu, M.; Rocca, J.; Fontana, M.; Urena, A.; Arcondo, B.; Pradel, A. J. Alloys Compd., 2010, 495, 642-645.

67. Baudet, E.; Cardinaud, C.; Girard, A.; Rinnert, E.; Michel, K.; Bureau, B.; Nazabal, V. J. Non Cryst. Solids., 2016, 444, 64-72.

68. Veres, M.; Kozyukhin, S.; Ingram, A.; Nguyen, H.P.; Kudoyarova, V. Phys. Proc., 2013, 44, 82-90.

69. Khan, P.; Arinjoy, B.; Abin, J.; Vasant, S.; Uday, D.; Adarsh, K.V. Thin Solid Films., 2017, 621, 76-83.

70. Mukta, B.; Naik, P.; Panda, R.; Naik, R. Opt. Mater., 2017, 66, 616-622.

71. Kim, Y.; Choi, I. Thin Solid Films., 2019, 669, 351-354.
72. Knal, J.T.; Vinod, V.; Subrahmanyam, A.; Malar, P. Appl. Surf. Sci., 2017, 418, 216-224.

73. Kumar, D.S.; Ren, M.; Thomas, O.; Mallik, R.C.; Malar, P. Sol. Energy., 2018, 174, 422430.

74. Dong, Y.; Lu, X.; Shen, P.; Chen, Y.; Yue, F.; Xiang, P.; Sun, L.; Yang, P.; Chu, J. Mater. Sci. Semicond. Process., 2018, 84, 124-130.

75. Biren, P.; Ranjan, K.P.; Indrajit, M.; Ray, A. Vacuum., 2018, 158, 263-270.

76. Juskenas, R.; Zenius, M.; Raimondas, G.; Algirdas, S.; Giedrius, S.; Stase, K.; Audrius, D. J. Alloys Compd., 2018, 767, 345-352.

77. Lei, Q.; Xu, J.; Cai, W.; Xie, Z.; Yang, Y. Superlattices Microstruct., 2019, 126, 83-88.

78. Osama, A.; Zhe, C. Sol. Energy Mater. Sol. Cells., 2018, 176, 222-229.

79. Chayma, N.; Mehdi, S.; Yvan, C.; Najoua, K. Superlattices Microstruct., 2018, 124, 17-29.

80. Houda, T.;Bouloufa, A.; Marek, P.; Igalson, M. Mater. Sci. Semicond. Process., 2018, 88, 167-172.

81. Sampath, M.; Sankarasubramanian, K.; Archana, J.; Hayakawa, Y.; Ramamurthi, K.; Sethuraman, K. Mater. Sci. Semicond. Process., 2018, 87, 54-64.

82. Lugo, S.; Sanchez, Y.; Neuschitzer, M.; Xie, H.; Insignares, C.; Roca, V.; Pena, Y.; Saucedo, E. Thin Solid Films., 2015, 582, 74-78.

83. Liu, Y.; Zhang, X.; Li, Q.; Long, M.; Hou, J.; Na, Z.; Zhao, G.; Fang, Y. J. Alloys Compd., 2017, 695, 3146-3151.

84. Kim, Y., Choi, I. J. Korean Phys. Soc., 2017, 70, 281-285.

85. Mahesh, P.S.; Uma, V.G.; Umesh, P.S.; He, M.; Kim, J.; Gang, M.G.; Patil, P.S.; Moholkar, A.V.; Yun, J.H.; Kim, J. H. ACS Omega., 2017, 2, 9211-9220.

86. Huang, M.; Chen, D.H.;Wei, A.X.; Xiao, Z.M.; Liu, J.; Zhao, Y. Funct. Nanostruct. Proc., 2017, 2, 10-12. 